\section{OPEN ACCESS}

Edited by

Pedro A. Reche,

Complutense University of

Madrid, Spain

Reviewed by:

Robert Sinden,

Imperial College London, United Kingdom

Martino Bardelli,

University of Oxford, United Kingdom

*Correspondence:

Shigeto Yoshida

shigeto@p.kanazawa-u.ac.jp

Specialty section:

This article was submitted to Vaccines and Molecular Therapeutics,

a section of the journal

Frontiers in Immunology

Received: 17 June 2019

Accepted: 26 September 2019

Published: 15 October 2019

Citation:

Yusuf Y, Yoshii T, lyori M, Mizukami $H$,

Fukumoto S, Yamamoto DS,

Emran TB, Amelia F, Islam A, Syafira I and Yoshida S (2019) A Viral-Vectored Multi-Stage Malaria Vaccine Regimen

With Protective and

Transmission-Blocking Efficacies.

Front. Immunol. 10:2412

doi: 10.3389/fimmu.2019.02412

\title{
A Viral-Vectored Multi-Stage Malaria Vaccine Regimen With Protective and Transmission-Blocking Efficacies
}

\author{
Yenni Yusuf ${ }^{1,2}$, Tatsuya Yoshii ${ }^{1}$, Mitsuhiro lyori ${ }^{1}$, Hiroaki Mizukami ${ }^{3}$, Shinya Fukumoto ${ }^{4}$, \\ Daisuke S. Yamamoto ${ }^{5}$, Talha Bin Emran ${ }^{1}$, Fitri Amelia ${ }^{1}$, Ashekul Islam ${ }^{1}$, Intan Syafira ${ }^{1}$ \\ and Shigeto Yoshida ${ }^{1 *}$
}

\begin{abstract}
${ }^{1}$ Laboratory of Vaccinology and Applied Immunology, Kanazawa University School of Pharmacy, Kanazawa University, Kanazawa, Japan, ${ }^{2}$ Department of Parasitology, Faculty of Medicine, University of Hasanuddin, Makassar, Indonesia, ${ }^{3}$ Division of Genetics Therapeutics, Centre for Molecular Medicine, Jichi Medical University, Shimotsuke, Japan, ${ }^{4}$ National Research Centre for Protozoan Diseases, Obihiro University of Agriculture and Veterinary Medicine, Obihiro, Japan, ${ }^{5}$ Division of Medical Zoology, Department of Infection and Immunity, Jichi Medical University, Shimotsuke, Japan
\end{abstract}

Malaria parasites undergo several stages in their complex lifecycle. To achieve reductions in both the individual disease burden and malaria transmission within communities, a multi-stage malaria vaccine with high effectiveness and durability is a more efficacious strategy compared with a single-stage vaccine. Here, we generated viral-vectored vaccines based on human adenovirus type 5 (AdHu5) and adeno-associated virus serotype 1 (AAV1) expressing a fusion protein of the pre-erythrocytic stage Plasmodium falciparum circumsporozoite protein (PfCSP) and the transmission-blocking sexual stage P25 protein (Pfs25). A two-dose heterologous AdHu5-prime/AAV1-boost immunization regimen proved to be highly effective for both full protection and transmission-blocking activity against transgenic $P$. berghei parasites expressing the corresponding $P$. falciparum antigens in mice. Remarkably, the immunization regimen induced antibody responses to both PfCSP and Pfs25 for over 9 months after the boosting and also maintained high levels of transmission-reducing activity (TRA: $>99 \%$ ) during that period, as evaluated by a direct feeding assay. If similar efficacies on $P$. falciparum can be shown following vaccination of humans, we propose that this multi-stage malaria vaccine regimen will be a powerful tool for malaria control, providing greater overall protection and cost-effectiveness than single-stage vaccines.

Keywords: multi-stage malaria vaccine, transmission-blocking, Plasmodium falciparum circumsporozoite protein, Pfs25, human adenovirus serotype 5 , adeno-associated virus

\section{INTRODUCTION}

Malaria in humans is caused by Plasmodium spp. parasites, which undergo a complex lifecycle (1). The parasites are transmitted by Anopheles mosquitoes via the injection, during a bloodmeal, of sporozoites into the subcutaneous tissue, from which the sporozoites migrate to the liver and invade hepatocytes, then mature into schizont-containing merozoites that subsequently invade red blood cells, commencing the erythrocytic cycle. Some merozoites differentiate into gametocytes, which can be ingested by female mosquitoes, inside which they recombine into ookinetes that develop into oocysts in the mosquito midgut. The oocysts contain many sporozoites 
that migrate to the salivary gland and repeat the cycle in the next host. Throughout their lifecycle, the antigenic characteristics of the parasites change, the majority are not expressed at all stages; consequently, malaria vaccines specifically target different stages: pre-erythrocytic stage, erythrocytic stage, and sexual stage (2). The pre-erythrocytic stage is a prime target for intervention efforts because immunity against this stage is sterilizing; it prevents sporozoites from invading hepatocytes and/or it inhibits the development of liver-stage parasites into merozoites, thus eventually precluding the development of disease and the transmission of malaria $(2,3)$. This stage is the target of the RTS,S/AS01 vaccine, currently the most advanced malaria vaccine candidate, which acts through the induction of high levels of both anti-circumsporozoite (CSP) antibodies and CSP-specific $\mathrm{CD}^{+} \mathrm{T}$ cells, with a greater role being attributed to the antibody (4). However, in recent years, there has been an increased focus on the development of vaccines capable of breaking the cycle of Plasmodium by targeting the parasite sexual stages [transmission-blocking vaccines (TBV)], some of which have entered clinical trials (5-7). Both vaccine types, the preerythrocytic vaccines (PEV), and TBV, are categorized as vaccines that interrupt malaria transmission (VIMT) to support malaria elimination (8). Malaria vaccines are considered amongst the most important modalities for potential disease prevention and transmission reduction.

In 2013, the World Health Organization (WHO) malaria vaccine roadmap set two strategic goals to be met by 2030: (1) the development of vaccines that are highly efficacious in preventing clinical malaria and (2) the development of vaccines that prevent transmission, to accelerate malaria parasite elimination (7). An efficacious vaccine must either be completely effective against a stage, by eliminating over time the parasite or dramatically reducing parasite numbers, or else target multiple stages of the parasite lifecycle (2). Since such an effective vaccine is not yet available, the combination of partially effective vaccines that target different parasite stages provides another powerful way to achieve the WHO goals. A recent study demonstrated that partially efficacious interventions separately targeting the pre-erythrocytic and sexual stages have a synergistic impact in eliminating malaria from a population over multiple generations (9). Several studies have investigated the application of a mixture or co-administration of vaccines targeting different stages (1014), including the most recent combination of RTS,S/AS01 and the current leading TBV candidate Pfs25-IMX313 (15), with some promising results.

Notably, a multi-stage vaccine, a vaccine targeting different stages of parasite life cycle in one construct, may provide a more cost-effective solution than a vaccination approach that uses mixtures of multiple single-stage vaccines. In addition, this approach may also be more convenient for the vaccine recipients than the co-administration of multiple vaccines. Unfortunately, there has not been much success yet in the development of such a multi-stage malaria vaccine (5). Several studies investigating potential multi-stage malaria vaccines found generally poor antibody responses and limited efficacies (16-19).

The progress of viral-vectored vaccines for malaria through the clinical development pathway has accelerated considerably
(20). Very recently, our group generated a series of human adenovirus 5 (AdHu5) and adeno-associated virus serotype 1 (AAV1) expressing either $P$. falciparum pre-erythrocytic PfCSP or sexual stage Pfs 25 antigen. Heterologous two-dose immunization with an AdHu5-prime and AAV1-boost elicited a high level of protection against sporozoite challenge and excellent TB activity with sustained high-titer antibody responses (21). Using the same platform, the present study aimed to develop a potent multi-stage malaria vaccine to effectively induce durable immunity for both protection and TB, which lasts for at least one transmission season ( $\sim 6$ months). In pursuit of this aim, we generated AdHu5- and AAV1-vectored multi-stage vaccines harboring "the $p f s 25-p f c s p$ fusion gene." Transgenic $P$. berghei expressing either the $p f c s p$ or $p f s 25$ genes were used to evaluate the protective and TB efficacies of this candidate multi-stage vaccine in a murine model. Our results demonstrate that the multi-stage vaccine regimen has the potential to fulfill the landmark goals of the malaria vaccine technology roadmap by achieving sterile protection and long-term TB efficacy.

\section{MATERIALS AND METHODS}

\section{Ethics Statement}

All animal care and handling procedures were approved by the Animal Care and Use Committee of Kanazawa University (No. AP-163700) and in accordance with the Guidelines for Animal Care and Use prepared by Jichi Medical University (No. 09193). All efforts were made to minimize suffering in the animals.

\section{Parasites and Animals}

The transgenic P. berghei Pfs25DR3 (PbPfs25DR3) that was used for TB assays and the ookinete immunofluorescence assays (IFAs) was kindly donated by A. Blagborough from Imperial College London (22). The transgenic P. berghei expressing PfCSP (PfCSP-Tc/Pb) that was used for the IFAs and the protective efficacy study was described previously (21-23). Both transgenic parasites were maintained in the Laboratory of Vaccinology and Applied Immunology, Kanazawa University. Anopheles stephensi mosquitoes (SDA 500 strain) were infected with the transgenic parasites by allowing them to feed on parasite-infected 6-weekold ddY mice. All other animal experiments used 6-week-old $\mathrm{BALB} / \mathrm{c}$ mice.

\section{Viral Vector Construction}

For the generation of AdHu5-Pfs25-PfCSP, the gene encoding Pfs25 and the Gly6Ser hinge was excised from pUC57-SimplesPfs25-hinge (21) by digestion with EcoRI/MefI and then inserted into the EcoRI site of pENTR-D-sPfCSP2-G2-sWPRE (23) to construct pENTR-D-fusion. Pfs25-PfCSP was excised by digestion of pENTR-D-sPfs25-sPfCSP-WPRE with EcoRI/XmaI and then inserted into the EcoRI/XmaI sites of pENTR-CAGsPfCSP2-G2-sWPRE (21) to construct pENTR-CAG-sPfs25sPfCSP2-G2-sWPRE, which was subsequently cloned into the shuttle vector pAd/PL-DEST (Invitrogen, Carlsbad, CA, USA) using a Gateway cloning technology. The adenovirus was purified and titrated as described previously (24). For the generation of AAV1-Pfs25-PfCSP, the gene cassette encoding the fusion 
Pfs25-PfCSP was excised from pENTR-CAG-sPfs25-sPfCSP2G2-sWPRE by digestion with KpnI and XhoI and then inserted into the KpnI and XhoI sites of pAAV-CMV-sPfs25 (21). The resulting plasmid, pAAV-CMV-sPfs25-sPfCSP2, was used to generate AAV1-Pfs25-PfCSP in HEK293 cells as described previously (25).

\section{Immunoblotting}

HEK293 cells were infected with the AdHu5 vaccine at a Multiplicity Of Infection (MOI) of 10 or with the AAV1 vaccine at a MOI of $10^{6}$. Cell lysates were collected using Laemmli buffer at $48 \mathrm{~h}$ post-infection and subjected to immunoblotting as described previously (24). The cell lysates were electrophoresed on $10 \%$ sodium dodecyl sulfate polyacrylamide (SDS-PAGE) gels under reducing conditions for probing with the antiPfCSP monoclonal antibody $(\mathrm{mAb}) 2 \mathrm{~A} 10$ or under non-reducing conditions for probing with anti-Pfs $25 \mathrm{mAb} 4 \mathrm{~B} 7$. Each blot was visualized using an Odyssey infrared imager (LI-COR, Lincoln, NE, USA).

\section{Immunofluorescence Assay}

IFAs to assess protein expression were performed as described previously (21). HEK293 cells were infected with the AdHu5 vaccine or AAV1 vaccine on an eight-well-chamber slide at a MOI of 10 or $10^{5}$, respectively. Cells were fixed with $100 \%$ methanol (permeabilized) or $4 \%$ paraformaldehyde (nonpermeabilized) for $30 \mathrm{~min}$ at $24 \mathrm{~h}$ post-infection. To visualize the expression of the antigen, the cells were incubated for $1 \mathrm{~h}$ at room temperature with Alexa-Fluor-488-conjugated 2A10 and Alexa-Fluor-596-conjugated 4B7, each diluted 1:100.

For IFAs of sporozoites and ookinetes, the sporozoites were isolated from mosquito salivary glands and then fixed with acetone/methanol (1:1) on glass slides, while the ookinetes were isolated from infected blood culture and then fixed with $4 \%$ paraformaldehyde. After blocking with $10 \%$ normal goat serum, slides were incubated with sera from immunized mice (1:80 dilution) for $1 \mathrm{~h}$, followed by incubation with FITC-conjugated goat anti-mouse IgG for $1 \mathrm{~h}$. For positive controls, sporozoites and ookinetes were stained with AlexaFluor 596-conjugated $2 \mathrm{~A} 10$ and $4 \mathrm{~B} 7$, respectively. In all IFA experiments, VECTASHIELD ${ }^{\circledR}$ containing 4', 6-diamidino-2phenylindole (DAPI) was used for nuclear staining, and a BZX710 fluorescence microscope (Keyence Corp, Tokyo, Japan) was used for image acquisition.

\section{Immunization}

All vaccines were administered intramuscularly in $100 \mu \mathrm{l}$ of phosphate-buffered saline (PBS). AdHu5 vaccines were administered at a dose of $5 \times 107$ plaque-forming units (PFU) as a prime, whereas AAV1 vaccines were administered at a dose of 1,011 vg per mouse as a boost. Immunization was performed with a 6-week interval between the prime and boost. For the vaccine mixture regimen, the same doses of AdHu5-PfCSP and AdHu5-Pfs25 $(5 \times 107 \mathrm{PFU})$ were mixed in a syringe as a prime, and the same doses of AAV1-PfCSP and AAV1-Pfs25 (1,011 vg) were mixed as a boost. Negative control animals were injected with either PBS or luciferase-expressing viruses (AdHu5-Lucprime/AAV1-Luc-boost).

\section{ELISA}

PfCSP- or Pfs25-specific antibody (Ab) levels were quantified by ELISA as described previously $(21,24)$. The PfCSP antigen was produced using an Escherichia coli expression system. The Pfs 25 antigen was produced using a wheat germ cell-free (WGCF) protein expression system (CellFree Sciences, Matsuyama, Japan) (26). Sera from immunized mice were collected from tail vein blood samples 1 day before boost and 1 day before challenge, or weekly up to 280 days post-boost for monitoring. Pre-coated Costar ${ }^{\circledR}$ EIA/RIA polystyrene plates (Corning Inc., NY, USA) with $400 \mathrm{ng} /$ well of PfCSP or $200 \mathrm{ng} /$ well of Pfs 25 were blocked with $1 \%$ bovine serum albumin (BSA) in PBS and then incubated with serially diluted sera samples, as well as with negative and positive controls (mAb $2 \mathrm{~A} 10$ or $\mathrm{mAb} 4 \mathrm{~B} 7$, respectively). An antimouse IgG conjugated with horseradish peroxidase (HRP) (BioRad lab, Inc., Tokyo, Japan) was used as the secondary Ab. The endpoint titer is expressed as the reciprocal of the last dilution that gave an optical density at $414 \mathrm{~nm}$ of $0.15 \mathrm{U}$ above the values of the negative controls $(<0.1)$. All mice used in our experiments were seronegative before immunization.

\section{Parasite Challenge Test}

Mice were intravenously challenged with $\mathrm{PfCSP}-\mathrm{Tc} / \mathrm{Pb}$ sporozoites resuspended in RPMI 1640 media (Gibco, Life Technologies, Tokyo, Japan). Sporozoites were prepared as described previously (27). Each mouse was injected via the tail vein with $100 \mu \mathrm{l}$ of media containing 500 sporozoites. Infection was monitored from day 4 to 14 by Giemsa staining of thin blood smears obtained from the tail. Protection was defined as the complete absence of blood-stage parasitemia on day 14 post-challenge. The time required to reach $1 \%$ parasitemia was determined as described previously (28).

\section{TB Assays}

TB was assessed using direct feeding assays (DFAs) as described previously (21). At 35 or 287 days after boost, mice were treated with phenylhydrazine and then infected intraperitoneally with $10^{6}$ PbPfs25DR3-parasitized red blood cells (pRBCs) 3 days later. At 3 days post-infection, at least 50 starved A. stephensi mosquitoes were allowed to feed on each infected mouse. At 5-6h post-feeding, any unfed mosquitoes were removed. Mosquitoes were then maintained on fructose $[8 \%(\mathrm{w} / \mathrm{v})$ fructose, $0.05 \%(\mathrm{w} / \mathrm{v}) p$-aminobenzoic acid] at $19-22^{\circ} \mathrm{C}$ and $50-80 \%$ relative humidity. On day $10-12$ post-feeding, the mosquito midguts were dissected, and oocyst prevalence and intensity were recorded. For each mouse, the number of oocysts was counted, and the mean oocyst intensity was calculated. For inhibition calculations, these numbers were compared with those of mice immunized with the AdHu5-AAV1 Luc control. Percent (\%) inhibition of mean oocyst intensity (transmission-reducing activity; TRA) was calculated as follows: $100 \times[1-$ (mean number of oocysts in the test group/mean number of oocysts in the control groups)]. Similarly, the \% inhibition of oocyst prevalence (transmission-blocking activity; TBA) was evaluated as: $100 \times[1-$ (proportion of mosquitoes with any oocysts in the test group/proportion of mosquitoes with any oocysts in the control groups)] (29). 


\section{Statistical Analysis}

For all statistical analyses, GraphPad Prism version 7.0 for Mac OS was used. Depending on the data distribution, a Student's $t$-test or Mann-Whitney rank test was used for comparing two groups. All ELISA end-point titers were $\log _{10}$ transformed before analysis. The proportion of mice not reaching $1 \%$ parasitemia was analyzed using a Kaplan-Meier log-rank (Mantel-Cox) test. The significance of TRA and TBA was assessed using the Mann-Whitney $U$-test and Fisher's exact probability test, respectively. A $p<0.05$ was considered statistically significant.

\section{RESULTS}

\section{Both AdHu5 and AAV1 Vaccines Expressed the Pfs25-PfCSP Fusion Antigen on the Surface of Mammalian Cells in vitro}

The $p f s 25-p f c s p$ fusion gene was linked by a sequence encoding a flexible hinge peptide (Gly ${ }_{6}$ Ser) between the $p f c s p$ and $p f s 25$ genes, and its expression was driven by the CAG and CMV promoter in AdHu5 and AAV1, respectively (Figure 1A). In the previous study, we reported that $\mathrm{Pfs} 25$ reacted with $\mathrm{mAb} 4 \mathrm{~B} 7$ in a ladder of bands with relative $\mathrm{Mr}$ of $33-48 \mathrm{kDa}$ due to posttranslational modifications because there are two potential $\mathrm{N}$ linked glycosylation sites in the predicted amino acid sequence of
Pfs25 (21). Similarly, the present study revealed that the Pfs25PfCSP fusion protein in the cells infected with AdHu5 (MOI $=10$, lane 1$)$ or AAV1 $\left(\mathrm{MOI}=10^{6}\right.$, lane 2$)$ reacted with both $\mathrm{mAb} 2 \mathrm{~A} 10$ (anti-PfCSP) and 4B7 (anti-Pfs25) in a ladder of bands with relative $M r$ of $80-100 \mathrm{kDa}$ (Figure 1B). In addition, there were several cleaves of the fusion protein causing the presence of a single band of PfCSP with relative $M r$ of $53 \mathrm{kDa}$ in the blot of protein with anti-PfCSP (Figure 1B, left panel). An IFA analysis demonstrated that both Pfs25 and PfCSP epitopes were expressed both in the cytoplasm and on the surface of infected cells (Figures 1C,D). Since the mAb $4 \mathrm{~B} 7$ recognizes a conformation-dependent epitope of Pfs25, these results suggest that the Pfs25-PfCSP fusion antigen on the surface of the infected cells retain the three-dimensional structure of the native Pfs 25 protein, which is essential for the induction of antibodies with TB functionality (30).

\section{The AdHu5-AAV1 Pfs25-PfCSP Regimen Induces Potent and Durable Anti-PfCSP and Anti-Pfs25 Immune Responses}

To investigate the immunogenicity of the AdHu5-Pfs25-PfCSPprime/AAV1-Pfs25-PfCSP-boost heterologous regimen (AdHu5AAV1 Pfs25-PfCSP), first we determined the PfCSP- and Pfs25specific antibody responses induced by the fusion vaccines as compared with those induced by the mixture of corresponding
A

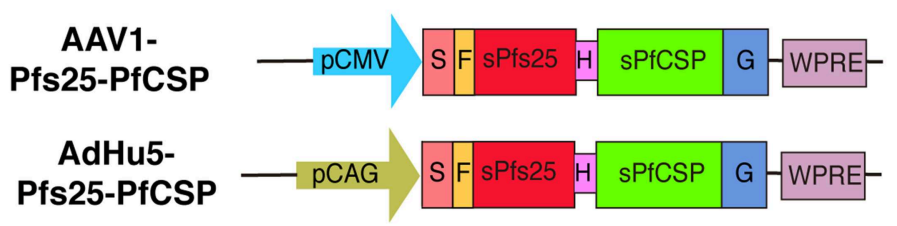

B
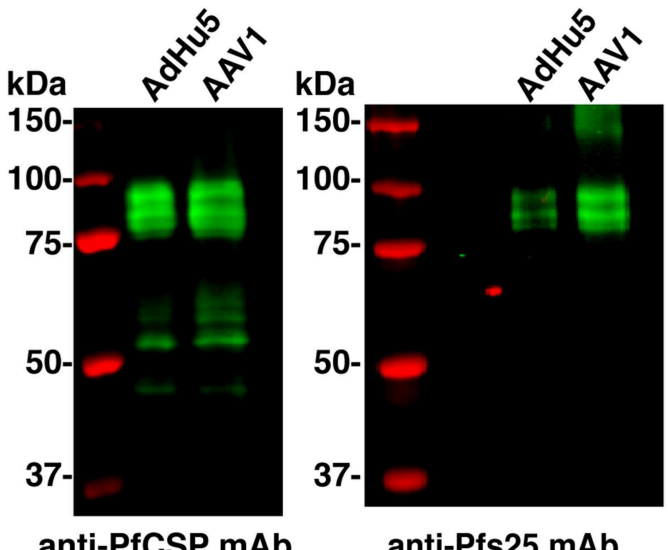

C

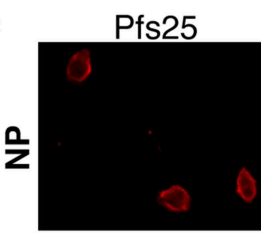

ฉ
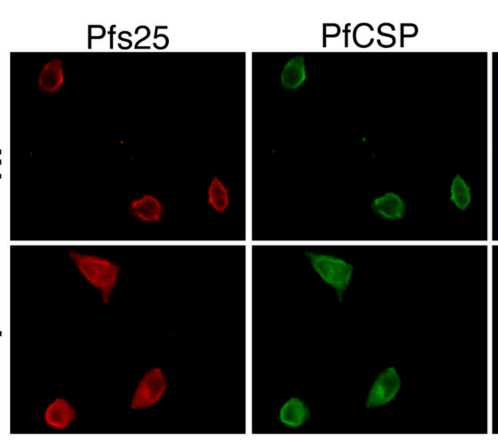

D

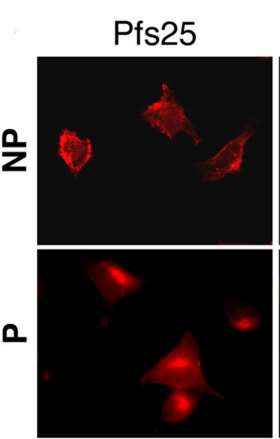

Merge + DAPI
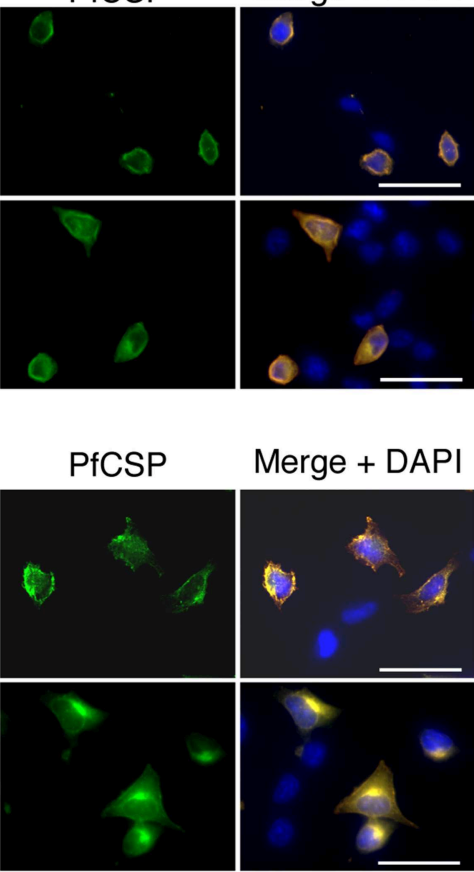

Merge + DAPI

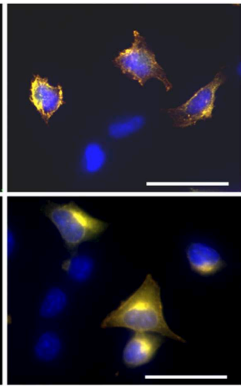

FIGURE 1 | Construction of viral-vectored vaccines. (A) Expression of the pfcsp and pfs25 gene cassettes was driven by the CMV promoter in AAV1 and by the CAG promoter in AdHu5. S, signal sequence; F, FLAG epitope tag; H, Hinge G6S; G, VSV-G transmembrane protein. (B) Analysis of the PfCSP and Pfs25 expression in HEK293T cells transduced with AdHu5-Pfs25-PfCSP (MOI =10) or AAV1-Pfs25-PfCSP $\left(\mathrm{MOI}=10^{6}\right)$. Cells were lysed and loaded onto a 10\% SDS-PAGE gel and immunoblotted with anti-PfCSP mAb $2 \mathrm{~A} 10$ and anti-Pfs25 mAb 4B7. (C,D) Localization of PfCSP and Pfs25 expression in mammalian cells after transduction with AdHu5-Pfs25-PfCSP (C) or with AAV1-Pfs25-PfCSP (D). After 24 h, the cells were fixed with paraformaldehyde (NP; non-permeabilized) or methanol (P; permeabilized) and blocked with 10\% normal goat serum. After being blocked, the cells were incubated with Alexa-Fluor-488-conjugated anti-PfCSP mAb (green) and Alexa-Fluor-594-conjugated anti-Pfs 25 mAb (red). Cell nuclei were visualized with DAPI (blue). Original magnification, $400 \times$. Scale bars $=50 \mu \mathrm{m}$. 

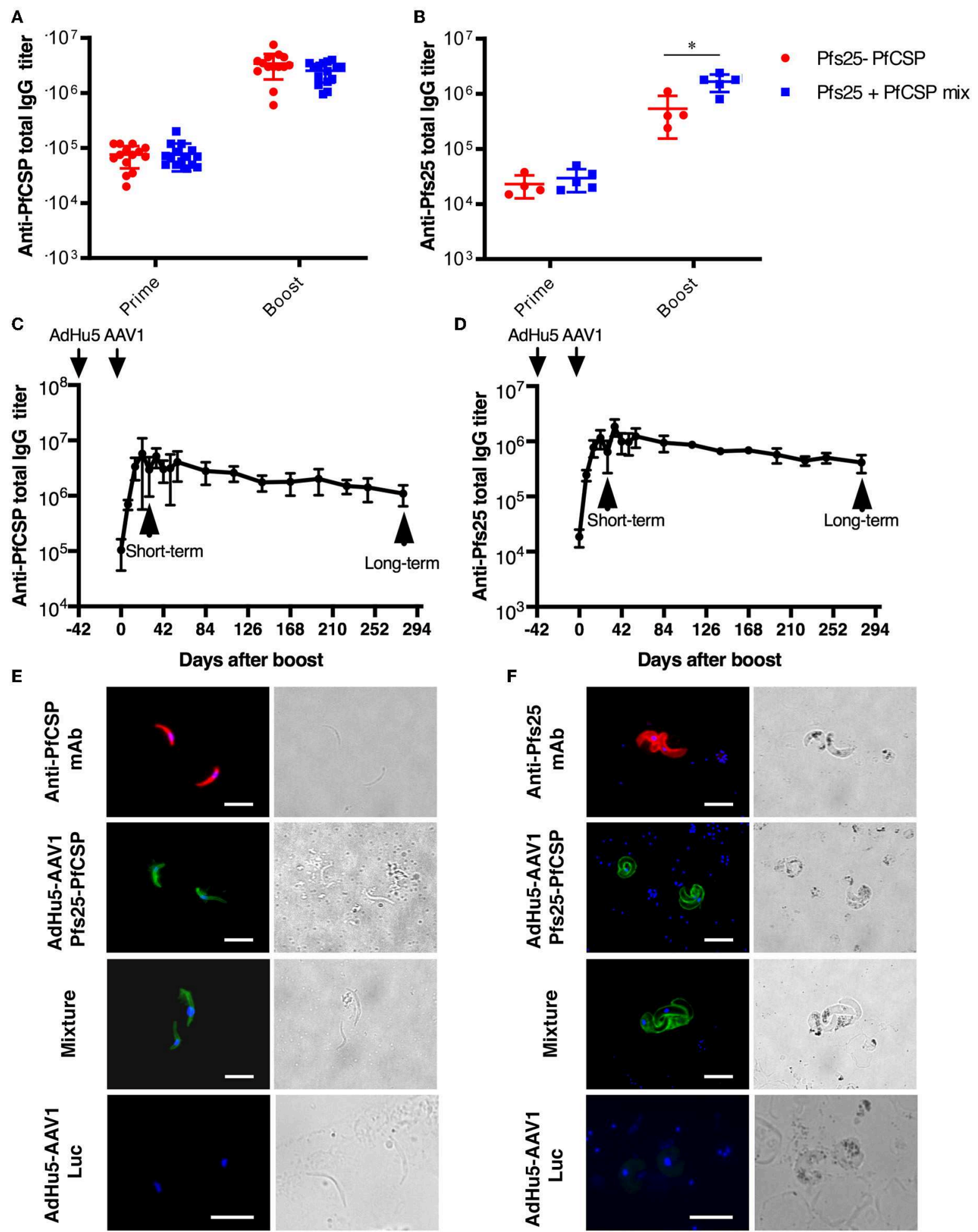

FIGURE 2 | Immunogenicity of the AdHu5-AAV1 Pfs25-PfCSP. (A,B) Comparison of antibody responses induced by the multi-antigen vaccine and the mixture of single-antigen vaccines. BALB/c mice were immunized with the indicated regimen. Individual sera were collected 4 weeks after boost, and antibody titers against PfCSP (A) and Pfs25 (B) were measured using ELISAs. The AdHu5-AAV1 Pfs25-PfCSP are labeled as "Pfs25-PfCSP"; the data from priming with a mixture of AdHu5-Pfs25 and AdHu5-PfCSP and boosting with a mixture of AAV1-Pfs25 and AAV1-PfCSP are labeled as "Pfs25+PfCSP mix." Each datapoint represents a single mouse. Horizontal lines indicate the means of antibody titers [ \pm standard deviation (SD)]. Differences between groups were assessed with a Mann-Whitney 
FIGURE 2 | U-test; * $p$ < 0.05. (C,D) Monitoring of antibody responses. BALB/c mice ( $n=5-10)$ were immunized with the AdHu5-AAV1 Pfs25-PfCSP at 6-week interval. Individual sera were collected 1 day before the boost, then weekly after the boost up to 280 days. Antibody titers against PfCSP (C) and Pfs25 (D) were measured using ELISAs. Data are presented as the mean \pm SD. (E,F) Reactivity of immune sera with the transgenic parasites. The transgenic PfCSP-Tc/Pb sporozoites (E) and ookinetes of Pfs25DR3 Pb (F) were fixed and incubated with sera from the immunized mice described in (A,B) and stained with FITC-conjugated goat anti-mouse IgG (green) for IFAs. The sporozoites and ookinetes were incubated with Alexa-594-conjugated 2A10 and 4B7, respectively, as positive controls. Cell nuclei were visualized with DAPI (blue). Original magnification, 1,000x. Scale bars $=10 \mu \mathrm{m}$.
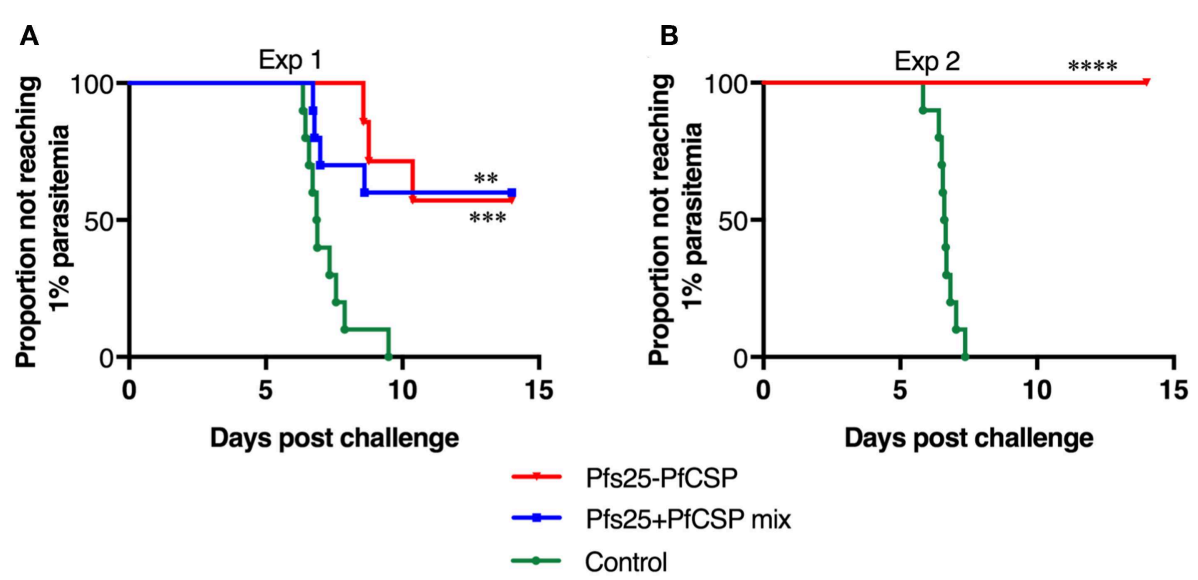

FIGURE 3 | Protective efficacy of the AdHu5-AAV1 Pfs25-PfCSP. BALB/c mice $(n=10)$ were immunized with the indicated regimen at 6-week interval. Four weeks after boost, the mice were challenged with an intravenous injection of 500 transgenic PfCSP-Tc/Pb sporozoites. Parasitemia was monitored for 3 consecutive days, starting from day 4 after challenge, and a model predicting the time to reach $1 \%$ parasitemia was generated. The absence of blood-stage parasites in the animals was confirmed on day 14 after challenge. The AdHu5-AAV1 Pfs25-PfCSP are labeled as "Pfs25-PfCSP" (A,B); data from mice primed with a mixture of AdHu5-Pfs25 and AdHu5-PfCSP and boosted with a mixture of AAV1-Pfs25 and AAV1-PfCSP are labeled as "Pfs25+PfCSP mix" (A). The statistical analysis was performed by generating Kaplan-Meier survival curves, and $p$-values were calculated with a Kaplan-Meier log-rank (Mantel-Cox) test. ${ }^{* \star * *} p<0.0001$, ${ }^{\star \star \star} p<0.001$, and ${ }^{* \star} p<0.01$ as compared with control groups.

single-antigen vaccines. Mice were immunized with a 6-week interval between the prime and boost $(n=15)$. Our preliminary studies revealed that mice immunized with the adenovirusprime/AAV-boost regimen achieved peak antibody titer at 4 weeks after boost. Therefore, at 4 weeks post-boost, sera of the immunized mice were collected for ELISA ( $n=14-15$ for PfCSP analysis; $n=4-5$ for Pfs 25 analysis). Both anti-PfCSP and anti-Pfs25 IgG titers were induced by the AdHu5-AAV1 Pfs25-PfCSP. Although the anti-Pfs25 IgG titer in the fusion group was slightly lower after boost compared with that in the mixture group $(537,500$ vs. 1,660,000) (Figure 2, the anti-PfCSP IgG titers were comparable between both groups $(3,439,286$ vs. $2,536,667$ ) (Figure 2A). Antibody monitoring of the animals immunized with the fusion vaccines showed that the IgG titers against both PfCSP and Pfs 25 remained high after 280 days (Figures 2C,D). The IFA results demonstrated that immune sera from mice vaccinated with the fusion vaccines reacted with the transgenic sporozoites (Figure 2E) and ookinetes (Figure 2F), at levels comparable to those observed for the immune sera from mice vaccinated with the mixture of single-antigen vaccines.

\section{The AdHu5-AAV1 Pfs25-PfCSP Confers Complete Protection Against Transgenic $P$. berghei Expressing PfCSP}

To assess the protective efficacy of the AdHu5-AAV1 Pfs25PfCSP, immunized mice were challenged with $\mathrm{PfCSP}-\mathrm{Tc} / \mathrm{Pb}$ sporozoites at 4 weeks post-boost, and the presence of blood infection was evaluated up to 14 days post-challenge. For comparison, the mice immunized with the mixture regimen were challenged at the same time. The fusion vaccine regimen conferred $57 \%$ protection, which is comparable with that of the mixture regimen (60\%) (Figure 3A). In another challenge experiment evaluating protective efficacy of the fusion vaccine only, the protection level reached 100\% (Figure 3B). Since the difference of mean antibody titer between both fusion groups was not statistically significant $(3,365,000$ vs. $3,775,000)$, we assumed that the variability in the protection level between the two experiments was due to the high variability of the intravenous sporozoite challenge itself as described by Leitner et al. (31).

\section{The AdHu5-AAV1 Pfs25-PfCSP Elicits a Durable TB Efficacy, Lasting at Least 287 Days}

It is widely accepted that the transmission blocking efficacy relates directly to the anti-Pfs25 Ab titer (32); thus, we expected the AdHu5-AAV1 Pfs25-PfCSP to have a high TB efficacy. To evaluate the functional activity of the anti-Pfs 25 Ab induced by AdHu5-AAV1 Pfs25-PfCSP, we assessed the TB efficacies at 35 days (short-term) and 287 days (long-term) after boost by performing DFAs. Groups of five mice were infected intraperitoneally with $10^{6}$ PbPfs25DR3-pRBCs. At 3 days after infection, three of the five mice in each group 


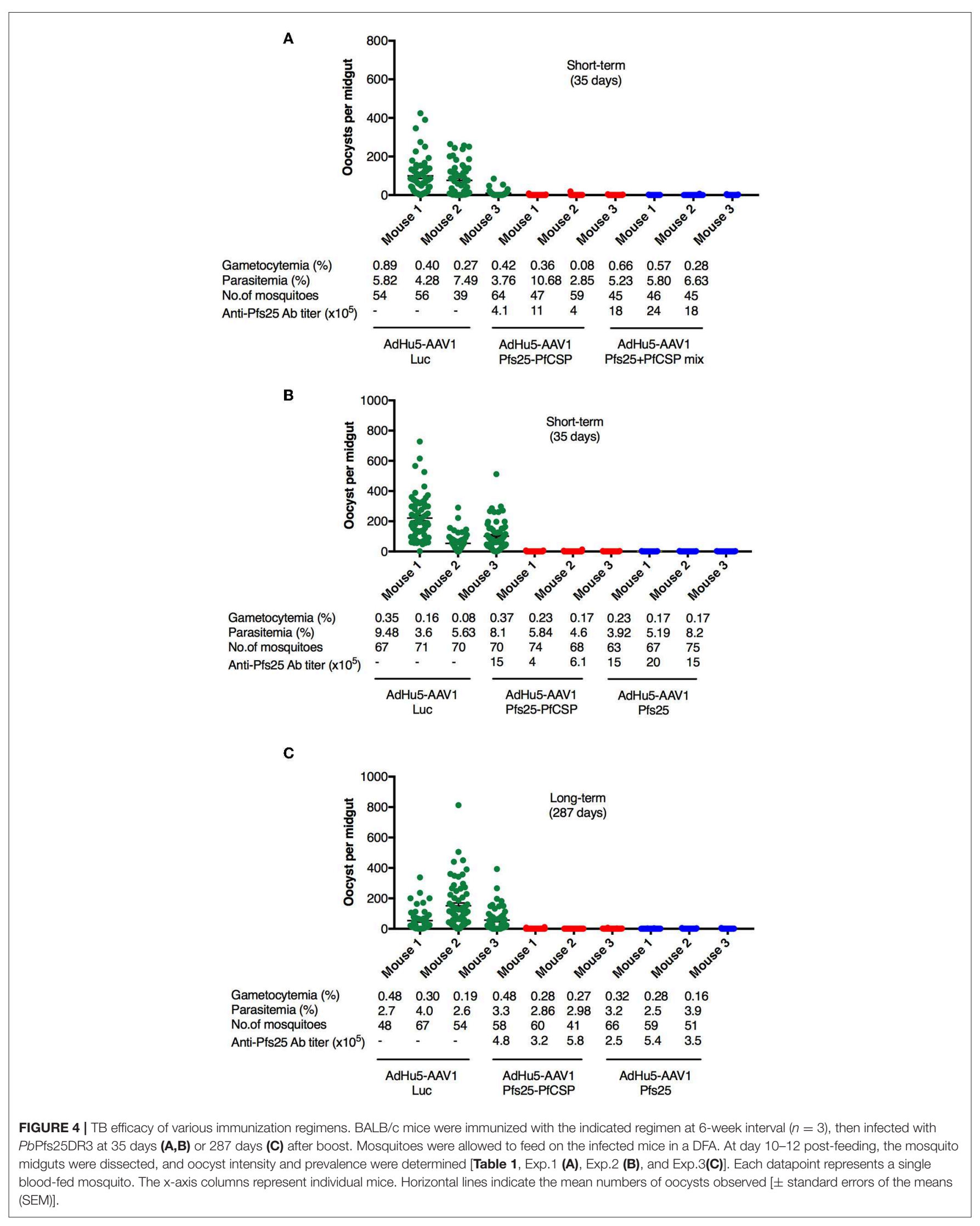


TABLE 1 | Transmission-blocking activity of the AdHu5-Pfs25-PfCSP prime/AAV1-Pfs25-PfCSP boost immunization regimen.

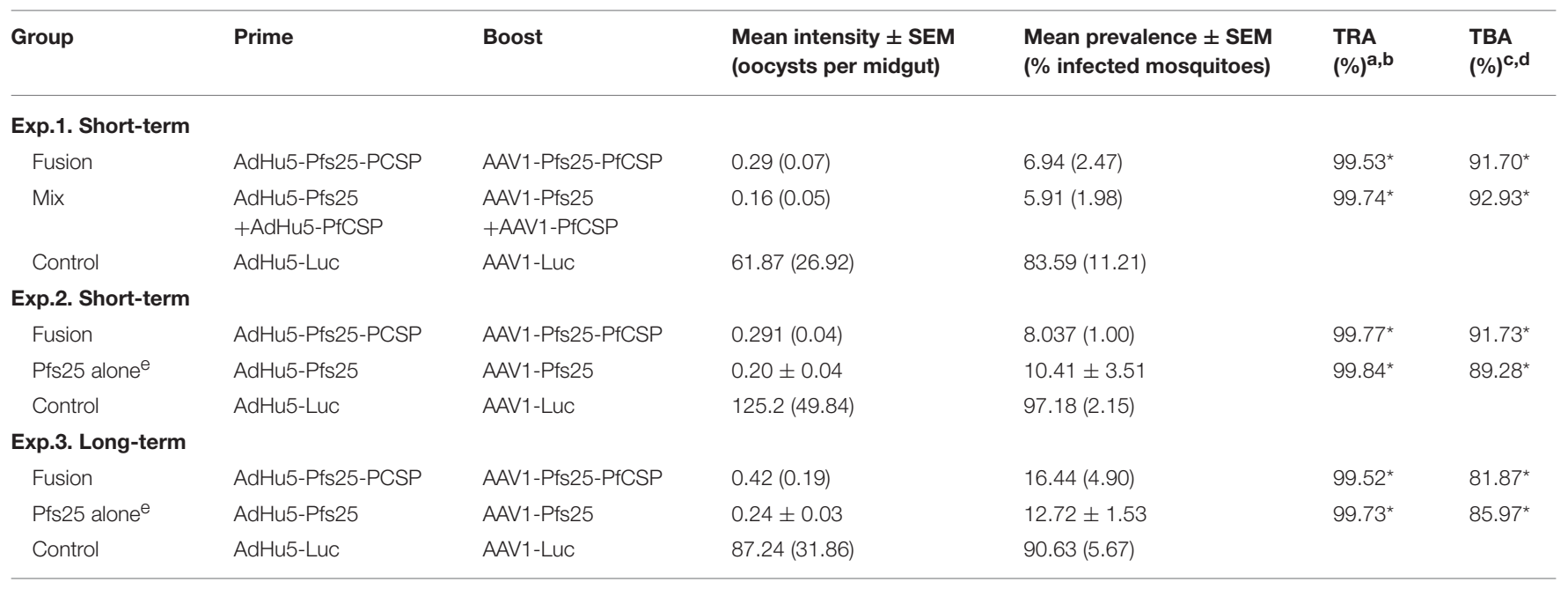

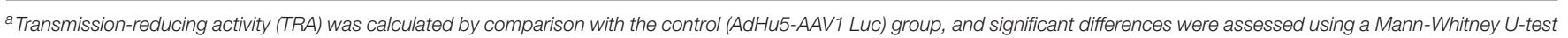
$\left({ }^{*} p<0.0001\right)$.

${ }^{b}$ No significant difference between the TRA of Pfs25-PfCSP and Pfs25+PfCSP mix ( $\left.p=0.6720\right)$ in Exp. 1.

${ }^{c}$ Transmission-blocking activity (TBA) was calculated by comparison with the control group, and significant differences were assessed using a Fisher's exact probability test ( $\left.p<0.0001\right)$.

${ }^{d}$ No significant difference between the TBA of Pfs25-PfCSP and Pfs25+PfCSP mix $(p=0.8171)$ in Exp. 1.

"These results have been previously reported in (21).

were chosen for DFA based on their parasitemia ( $>2 \%)$ and gametocytemia (>0.05\%) (Figure 4). A. stephensi mosquitoes were allowed to feed on each infected mouse, and the oocyst intensity and prevalence were recorded at 10-12 days post-feeding. Reductions in intensity and prevalence in the immunized mice were calculated with respect to the AdHu5AAV1 Luc-immunized controls.

First, we evaluated the short-term TB efficacy of the fusion vaccines as compared with the mixture of single-antigen vaccines (Table 1, Exp.1, Figure 4A). In this experiment, mosquitoes that fed on the three control mice displayed an average intensity of 61.87 oocysts/midgut. Following AdHu5-AAV1 Pfs25-PfCSP immunization, the mean intensity was only 0.29 oocysts/midgut, corresponding to a reduction (referred to as the TRA) of 99.53\% ( $p<0.0001)$, compared with the mean intensity of 0.16 oocysts/midgut in the mixture group (TRA of $99.74 \%$, $p<0.0001)$. Correspondingly, the mean infection prevalence was reduced from 83.59 to $6.94 \%$ and $5.91 \%$ in the AdHu5AAV1 Pfs25-PfCSP group and the mixture group, respectively, achieving significant reductions (referred to as the TBA) of 91.70\% $(p<0.0001)$ and $92.93 \%(p<0.0001)$. These results demonstrate that although there was a slight difference in the anti-Pfs 25 IgG titers between the fusion vaccine group and the mixture group, there were no significant differences in the TRA or TBA between these groups $(p=0.67$ and $p=$ 0.82 , respectively).

Next, to re-confirm the TB efficacy of the AdHu5-AAV1 Pfs25-PfCSP, we performed another short-term study (Table 1, Exp.2, Figure 4B). In this experiment, mosquitoes that fed on the three control mice displayed an average intensity of 125.17 oocysts/midgut and an infection prevalence of $97.18 \%$. The mean intensity was 0.29 oocysts/midgut in the immunized group, achieving a TRA of $99.77 \%(p<0.0001)$, and the mean infection prevalence was $8.04 \%$, achieving a TBA of $91.73 \%(p<0.0001)$. These results are comparable with those of Exp.1. In addition, there are no significant differences with our previous data on the short-term TRA and TBA of the AdHu5-Pfs25-prime/AAV1Pfs25-boost (AdHu5-AAV1 Pfs25) (21).

Finally, we evaluated the long-term TB efficacy of the AdHu5AAV1 Pfs25-PfCSP at 287 days after the booster injection (Table 1, Exp.3, Figure 4C). The experiment revealed that the TRA did not significantly decline over 287 days. The mean oocyst intensity in the immunized group was 0.42 oocysts/midgut (compared with 87.24 oocysts/midgut in the control group), achieving a TRA of $99.52 \%(p<0.0001)$, and the mean infection prevalence was $16.44 \%$ compared with the $90.63 \%$ of the control group, achieving a TBA of $81.87 \%(p<0.0001)$. This result similarly showed no significant differences with our previous data on the long-term TRA and TBA of the AdHu5-AAV1 Pfs25 (21).

Collectively, our data demonstrate that the AdHu5-AAV1 Pfs25-PfCSP is an effective multi-stage malaria vaccine, capable of inducing a high level of PfCSP- and Pfs25-specific Ab immune responses and achieving a high level of protective immunity and long-term TB immunity against the malaria parasite.

\section{DISCUSSION}

Here, we demonstrated that an immunization regime using AdHu5- and AAV1-based multi-stage malaria vaccines harboring a gene encoding the pre-erythrocytic antigen PfCSP fused with the mosquito-stage antigen Pfs 25 is protective and has durable TB efficacy. The AdHu5-AAV1 prime-boost approach evoked high levels of antibody titers against both antigens, which were sustained for at least one transmission season; this is one of the desired features of an ideal malaria vaccine (33). Remarkably, the administration 
of these vaccines in a prime-boost combination achieved both protection and $\mathrm{TB}$ immunity in a murine model, fulfilling the urgent need for an effective second-generation malaria vaccine that reduces transmission and incidence, rather than simply reducing the morbidity and mortality of the disease $(7,34)$. Moreover, it may help meet the strategic goals for 2030 set by the WHO malaria vaccine roadmap (7).

The Malaria Eradication Research Agenda Consultative Group on Vaccines (malERACGoV) (8) requires that a VIMT must primarily reduce malaria transmission. A highly effective PEV that prevents erythrocytic stage infection will obviously reduce transmission, but the current leading PEV candidate, RTS,S/AS01, confers only $36.3 \%$ protection $(35,36)$. However, it has been suggested that a successful TBV would ideally be combined with a vaccine that blocks sporozoite/liver stage development and/or asexual blood stage development (37). Hence, mixing a PEV and TBV together might provide a means of achieving an effective VIMT. In the present study, we selected the immunogen Pfs 25 to exert a TB effect because it was demonstrated that Pfs 25 may induce functional antibodies in humans that effectively inhibit malaria parasite development in mosquitoes $(38,39)$. We focused on a preerythrocytic antigen, rather than a blood-stage antigen, for use in conjunction with Pfs 25 because this combination was suggested as likely to be the most efficient in reducing malaria prevalence; a malaria model analysis of pathogen virulence evolution predicted that PEVs select for lower parasite virulence, while blood-stage vaccines select for higher parasite virulence, so a combination of these may increase the population-level benefits of vaccination (40).

The use of a multi-stage vaccine regimen may increase the adherence to a malaria elimination vaccination program because people will be more likely to go get vaccinated if the shot also provides personal protection from the disease. A recent study investigated the potential of mixing the RTS,S/AS01 with Pfs25-IMX313/AS01 in one formulation or co-administering both vaccines (15), and it found that the combination of these two vaccines elicited antibody titers against both PfCSP and Pfs25 that were similar to those elicited by the corresponding single-antigen vaccines. Using in vitro assays, it was also shown that this combination of vaccines exhibited similar functional activity in $\mathrm{TB}$ and sporozoite inhibition. Using in vivo assays, our results from mice immunized with the mixture of single-antigen formulations are in line with those of this previous study, in contrast with the studies that found immune interference or reduced efficacies for combinations of several other malaria vaccines (11, 41, 42).

Notably, mixing two or more vaccines in a single formulation can result in a higher vaccination cost. Thus, the development of a multi-stage vaccine harboring different antigens from different parasite stages may be the best way of reducing the vaccination cost. To address this issue, we employed the multistage AdHu5-AAV1 Pfs25-PfCSP and demonstrated that this regimen has a similar efficacy as the mixture of corresponding single-antigen formulations, in terms of both protection and
TB activity. Remarkably, the TB activity lasted for over 9 months, with the sustained high titer of antibodies against Pfs25 exceeding one transmission season. These results are in contrast with those from previous attempts at the development of multi-stage malaria vaccines, which generally demonstrated poor antibody responses (16-19). A similar conjugated vaccine with a successful induction of antibody responses against both CSP and Pfs 25 has been reported by only one group, Kubler-Kielb et al.; they used protein adjuvanted with alum (43) and did not evaluate the protective efficacy of the vaccine. Additionally, their study administered the vaccines via three doses with 2 -week intervals, whereas the number of doses in our study was reduced to only two separated by a longer interval.

One limitation of this study is that the long-term protective efficacy of the regimen has not been investigated. This feature, both on mice that have not been challenged before and on mice that were protected from a challenge, will be evaluated in our future work. However, even if the longterm protective efficacy is lower than the protective efficacy observed here, this regimen could still be a great tool for supporting the malaria elimination program because antisporozoite and anti-transmission interventions may act synergistically to accelerate malaria elimination efforts over multiple generations (9). Moreover, the additional TB immunity combined with the pre-erythrocytic immunity conferred by this multi-stage regimen is an improvement over the RTS,S/AS01 for a next-generation vaccine (44). Thus, we are currently planning further evaluation of this regimen in human trials.

\section{DATA AVAILABILITY STATEMENT}

The raw data supporting the conclusions of this manuscript will be made available by the authors, without undue reservation, to any qualified researcher.

\section{ETHICS STATEMENT}

The animal study was reviewed and approved by Animal Care and Use Committee of Kanazawa University, Guidelines for Animal Care and Use Jichi Medical University. Written informed consent was obtained from the owners for the participation of their animals in this study.

\section{AUTHOR CONTRIBUTIONS}

YY, MI, and SY: study concept and design, and drafting the manuscript. YY, TY, MI, SF, DY, TE, FA, AI, IS, and SY: acquisition of data. YY, TY, MI, and SY: analyses and interpretation of data. MI and SY: critical revision of the manuscript for important intellectual content. YY and TY: statistical analyses. YY, TY, MI, HM, SF, DY, TE, FA, AI, IS, and SY: technical or material support. SY: study supervision. 


\section{FUNDING}

This work was supported by a Grant-in-Aid for Scientific Research (C) (JSPS KAKENHI grant number 18K06655) to MI; by a Grant-in-Aid for Scientific Research (B) (JSPS KAKENHI grant number 16H05815) and a Grant-in-Aid for Challenging Exploratory Research (JSPS KAKENHI grant number 18K19394) to SY; and by a thesis and dissertation fund by the Indonesia Endowment Fund for Education (LPDP) to YY.

\section{REFERENCES}

1. Igweh JC. Biology of malaria parasites. In: Okwa DO, editor. Malaria Parasites. Rijeka: InTech (2012). p. 11-36.

2. Doolan DL, Hoffman SL. Multi-gene vaccination against malaria: a multistage, multi-immune response approach. Parasitol Today. (1997) 13:171-8. doi: 10.1016/S0169-4758(97)01040-5

3. Doolan DL, Martinez-Alier N. Immune responses to preerythrocytic stages of malaria parasites. Curr Mol Med. (2006) 6:169-85. doi: 10.2174/156652406776055249

4. White MT, Bejon P, Olotu A, Griffin JT, Riley EM, Kester KE, et al. The Relationship between RTS,S vaccine-induced antibodies, CD4 ${ }^{+} \mathrm{T}$ cell responses and protection against Plasmodium falciparum infection. PLoS ONE. (2013) 8:e61395. doi: 10.1371/journal.pone.0061395

5. Birkett AJ. Building an effective malaria vaccine pipeline to address global needs. Vaccine. (2015) 33:7538-43. doi: 10.1016/j.vaccine.2015.09.111

6. World Health Organization. Malaria Vaccine Rainbow Tables. Available online at: http://www.who.int/vaccine_research/links/Rainbow/en/index. html (accessed December 20, 2018).

7. Moorthy VS, Newman RD, Okwo-Bele JM. Malaria vaccine technology roadmap. Lancet. (2013) 382:1700-1. doi: 10.1016/S0140-6736(13)62238-2

8. Birkett AJ, Moorthy VS, Loucq C, Chitnis CE, Kaslow DC. Malaria vaccine R\&D in the decade of vaccines: breakthroughs, challenges and opportunities. Vaccine. (2013) 31:B233-43. doi: 10.1016/j.vaccine.2013.02.040

9. Sherrard-Smith E, Sala KA, Betancourt M, Upton LM, Angrisano F, Morin MJ, et al. Synergy in anti-malarial pre-erythrocytic and transmissionblocking antibodies is achieved by reducing parasite density. Elife. (2018) 7:e35213. doi: 10.7554/eLife.35213

10. Forbes EK, Biswas S, Collins KA, Gilbert SC, Hill AV, Draper SJ. Combining liver- and blood-stage malaria viral-vectored vaccines: investigating mechanisms of $\mathrm{CD}^{+} \mathrm{T}$ cell interference. J Immunol. (2011) 187:3738-50. doi: 10.4049/jimmunol.1003783

11. Sheehy SH, Duncan CJA, Elias SC, Choudhary P, Biswas S, Halstead FD, et al. ChAd63-MVA-vectored blood-stage malaria vaccines targeting MSP1 and AMA1: assessment of efficacy against mosquito bite challenge in humans. Mol Ther. (2012) 20:2355-68. doi: 10.1038/mt.2012.223

12. Sedegah M, Tamminga C, McGrath S, House B, Ganeshan H, Lejano J, et al. Adenovirus 5-vectored P. falciparum vaccine expressing CSP and AMA1. Part A: safety and immunogenicity in seronegative adults. PLoS ONE. (2011) 6:e24586. doi: 10.1371/journal.pone.0024586

13. Tamminga C, Sedegah M, Regis D, Chuang I, Epstein JE, Spring M, et al. Adenovirus-5-Vectored P. falciparum vaccine expressing CSP and AMA1. Part B: safety, immunogenicity and protective efficacy of the CSP component. PLoS ONE. (2011) 6:e25868. doi: 10.1371/journal.pone.0025868

14. Chuang I, Sedegah M, Cicatelli S, Spring M, Polhemus M, Tamminga C, et al. DNA prime/adenovirus boost malaria vaccine encoding $P$. falciparum CSP and AMA1 induces sterile protection associated with cell-mediated immunity. PLoS ONE. (2013) 8:e55571. doi: 10.1371/journal.pone.0055571

15. Brod F, Miura K, Taylor I, Li Y, Marini A, Salman AM, et al. Combination of RTS,S and Pfs25-IMX313 induces a functional antibody response against malaria infection and transmission in mice. Front Immunol. (2018) 9:2780. doi: 10.3389/fimmu.2018.02780

16. Porter DW, Thompson FM, Berthoud TK, Hutchings CL, Andrews L, Biswas $\mathrm{S}$, et al. A human Phase I/IIa malaria challenge trial of a polyprotein malaria vaccine. Vaccine. (2011) 29:7514-22. doi: 10.1016/j.vaccine.2011.03.083

\section{ACKNOWLEDGMENTS}

The authors thank A. Blagborough for providing PbPfs25DR3 parasites and for kind assistance with the statistical analysis. The authors are grateful for the assistance of C. Seki and all members of the Laboratory of Vaccinology and Applied Immunology, Kanazawa University. The authors also thank Katie Oakley, Ph.D., from Edanz Group (www.edanzediting.com/ac) for editing a draft of this manuscript.

17. Tine JA, Lanar DE, Smith DM, Wellde BT, Schultheiss P, Ware LA, et al. NYVAC-Pf7: a poxvirus-vectored, multiantigen, multistage vaccine candidate for Plasmodium falciparum malaria. Infect Immun. (1996) 64:3833-44.

18. Ockenhouse CF, Sun PF, Lanar DE, Wellde BT, Hall BT, Kester K, et al. Phase I/IIa safety, immunogenicity, and efficacy trial of NYVAC-Pf7, a pox-vectored, multiantigen, multistage vaccine candidate for Plasmodium falciparum malaria. J Infect Dis. (1998) 177:1664-73. doi: 10.1086/515331

19. Spiegel H, Boes A, Kastilan R, Kapelski S, Edgue G, Beiss V, et al. The stagespecific in vitro efficacy of a malaria antigen cocktail provides valuable insights into the development of effective multi-stage vaccines. Biotechnol J. (2015) 10:1651-9. doi: 10.1002/biot.201500055

20. Ewer KJ, Sierra-Davidson K, Salman AM, Illingworth JJ, Draper SJ, Biswas S, et al. Progress with viral vectored malaria vaccines: a multistage approach involving "unnatural immunity". Vaccine. (2015) 33:744451. doi: 10.1016/j.vaccine.2015.09.094

21. Yusuf Y, Yoshii T, Iyori M, Yoshida K, Mizukami H, Fukumoto S, et al. Adenoassociated virus as an effective malaria booster vaccine following adenovirus priming. Front Immunol. (2019) 10:730. doi: 10.3389/fimmu.2019.00730

22. Goodman AL, Blagborough AM, Biswas S, Wu Y, Hill AV, Sinden RE, et al. A viral vectored prime-boost immunization regime targeting the malaria Pfs25 antigen induces transmission-blocking activity. PLoS ONE. (2011) 6:e29428. doi: 10.1371/journal.pone.0029428

23. Iyori M, Blagborough AM, Sala KA, Nishiura H, Takagi K, Yoshida S. Protective efficacy of an IL-12-expressing baculoviral malaria vaccine. Parasite Immunol. (2017) 39:e12498. doi: 10.1111/pim.12498

24. Yoshida K, Iyori M, Blagborough AM, Salman AM, Dulal P, Sala KA, et al. Adenovirus-prime and baculovirus-boost heterologous immunization achieves sterile protection against malaria sporozoite challenge in a murine model. Sci Rep. (2018) 8:3896. doi: 10.1038/s41598-018-21369-y

25. Xin KQ, Mizukami H, Urabe M, Toda Y, Shinoda K, Yoshida A, et al. Induction of robust immune responses against human immunodeficiency virus is supported by the inherent tropism of adeno-associated virus type 5 for dendritic cells. J Virol. (2006) 80:11899. doi: 10.1128/JVI.00890-06

26. Miura K, Takashima E, Deng B, Tullo G, Diouf A, Moretz SE, et al. Functional comparison of Plasmodium falciparum transmission-blocking vaccine candidates by the standard membrane-feeding assay. Infect Immun. (2013) 81:4377-82. doi: 10.1128/IAI.01056-13

27. Emran TB, Iyori M, Ono Y, Amelia F, Yusuf Y, Islam A, et al. Baculovirusinduced fast-acting innate immunity kills liver-stage Plasmodium. J Immunol. (2018) 201:2441-51. doi: 10.1101/320036

28. Epstein JE, Tewari K, Lyke KE, Sim BK, Billingsley PF, Laurens MB, et al. Live attenuated malaria vaccine designed to protect through hepatic CD8 T cell immunity. Science. (2011) 334:475-80. doi: 10.1126/science.1211548

29. Miura K, Swihart BJ, Deng B, Zhou L, Pham TP, Diouf A, et al. Transmissionblocking activity is determined by transmission-reducing activity and number of control oocysts in Plasmodium falciparum standard membrane-feeding assay. Vaccine. (2016) 34:4145-51. doi: 10.1016/j.vaccine.2016.06.066

30. Scally SW, McLeod B, Bosch A, Miura K, Liang Q, Carroll S, et al. Molecular definition of multiple sites of antibody inhibition of malaria transmission-blocking vaccine antigen Pfs25. Nat Commun. (2017) 8:1568. doi: 10.1038/s41467-017-01924-3

31. Leitner WW, Bergmann-Leitner ES, Angov E. Comparison of Plasmodium berghei challenge models for the evaluation of pre-erythrocytic malaria vaccines and their effect on perceived vaccine efficacy. Malaria J. (2010) 9:145. doi: 10.1186/1475-2875-9-145 
32. Miura K, Keister DB, Muratova OV, Sattabongkot J, Long CA, Saul A. Transmission-blocking activity induced by malaria vaccine candidates Pfs25/Pvs25 is a direct and predictable function of antibody titer. Malar J. (2007) 6:107. doi: 10.1186/1475-2875-6-107

33. Kappe SH, Vaughan AM, Boddey JA, Cowman AF. That was then but this is now: malaria research in the time of an eradication agenda. Science. (2010) 328:862. doi: 10.1126/science. 1184785

34. Menon V, Kapulu MC, Taylor I, Jewell K, Li Y, Hill F, et al. Assessment of antibodies induced by multivalent transmission-blocking malaria vaccines. Front Immunol. (2018) 8:1998. doi: 10.3389/fimmu.2017.01998

35. Olotu A, Fegan G, Wambua J, Nyangweso G, Leach A, Lievens M, et al. Seven-year efficacy of RTS,S/AS01 malaria vaccine among young African children. N Engl J Med. (2016) 374:2519-29. doi: 10.1056/NEJMoa15 15257

36. RTS, S Clinical Trials Partnership. Efficacy and safety of RTS,S/AS01 malaria vaccine with or without a booster dose in infants and children in Africa: final results of a phase 3, individually randomised, controlled trial. Lancet. (2015) 386:31-45. doi: 10.1016/S0140-6736(15)60721-8

37. Good MF, Yanow SK. A whole parasite transmission-blocking vaccine for malaria: an ignored strategy. Emerg Top Life Sci. (2017) 1:547. doi: 10.1042/ETLS20170117

38. Wu Y, Ellis RD, Shaffer D, Fontes E, Malkin EM, Mahanty S, et al. Phase 1 trial of malaria transmission blocking vaccine candidates Pfs 25 and Pvs25 formulated with montanide ISA 51. PLoS ONE. (2008) 3:e2636. doi: 10.1371/journal.pone.0002636

39. Saul A. Efficacy model for mosquito stage transmission blocking vaccines for malaria. Parasitology. (2008) 135:1497-506. doi: 10.1017/S0031182008000280

40. Gandon S, Mackinnon MJ, Nee S, Read AF. Imperfect vaccines and the evolution of pathogen virulence. Nature. (2001) 414:751. doi: 10.1038/414751a

41. Elias SC, Collins KA, Halstead FD, Choudhary P, Bliss CM, Ewer KJ, et al. Assessment of immune interference, antagonism, and diversion following human immunization with biallelic blood-stage malaria viralvectored vaccines and controlled malaria infection. J Immunol. (2013) 190:1135. doi: 10.4049/jimmunol.1201455

42. Bowyer G, Grobbelaar A, Rampling T, Venkatraman N, Morelle D, Ballou $\mathrm{RW}$, et al. CXCR3 ${ }^{(+)} \mathrm{T}$ follicular helper cells induced by co-administration of RTS,S/AS01B and viral-vectored vaccines are associated with reduced immunogenicity and efficacy against malaria. Front Immunol. (2018) 9:1660. doi: 10.3389/fimmu.2018.01660

43. Kubler-Kielb J, Majadly F, Biesova Z, Mocca CP, Guo C, Nussenzweig R, et al. A bicomponent Plasmodium falciparum investigational vaccine composed of protein-peptide conjugates. Proc. Natl. Acad. Sci. USA. (2010) 107:11727. doi: 10.1073/pnas.0913374107

44. Draper SJ, Sack BK, King CR, Nielsen CM, Rayner JC, Higgins MK, et al. Malaria vaccines: recent advances and new horizons. Cell Host Microbe. (2018) 24:43-56. doi: 10.1016/j.chom.2018.06.008

Conflict of Interest: SY, MI, and HM are named inventors on filed patents related to immunization with the AAV anti-malaria vaccines. These products have not been commercialized.

The remaining authors declare that the research was conducted in the absence of any commercial or financial relationships that could be construed as a potential conflict of interest.

Copyright (C) 2019 Yusuf, Yoshii, Iyori, Mizukami, Fukumoto, Yamamoto, Emran, Amelia, Islam, Syafira and Yoshida. This is an open-access article distributed under the terms of the Creative Commons Attribution License (CC BY). The use, distribution or reproduction in other forums is permitted, provided the original author(s) and the copyright owner(s) are credited and that the original publication in this journal is cited, in accordance with accepted academic practice. No use, distribution or reproduction is permitted which does not comply with these terms. 